C2008 IEEE. Personal use of this material is permitted. However, permission to reprint/republish this material for advertising or promotional purposes or for creating new collective works for resale or redistribution to servers or lists, or to reuse any copyrighted component of this work in other works must be obtained from the IEEE. 


\title{
A Work-in-Progress Platform for Solving Knowledge Sharing Issues in Organizations
}

\author{
Hai Dong, Farookh Khadeer Hussain, Elizabeth Chang \\ Digital Ecosystems and Business Intelligence institute \\ Curtin University of Technology \\ Perth, Australia \\ \{hai.dong, farookh.hussain, elizabeth.chang\}@cbs.curtin.edu.au
}

\begin{abstract}
In this paper, we propose a knowledge sharing platform based on the technology of ontology and agent, in order to solve the issues in the knowledge sharing activities within organizations. Based on the analysis of literature, the issues within current organizational knowledge sharing activities and knowledge sharing technologies are explored. By means of the literature analysis, we present a new definition of knowledge sharing in the context of organizational environment. Against these issues, our research objectives are delivered, and potential research effects are indicated. After that we choose a research approach and propose the conceptual model of our knowledge sharing platform. Finally we state the progress of our current research work, make conclusions and plan further works.
\end{abstract}

\section{INTRODUCTION}

Nowadays, knowledge management has become a crucial term in the IT industry [18]. Competitive organization can be seen as the generator and transformer of tacit and explicit knowledge [23]. The competition among enterprises has evolved to competing with knowledge stocks [21]. Thus, how to manage the knowledge is the vital issue for companies to achieve competitive advantages [20]. With the increasing interest on knowledge, we have entered an era of knowledge management, and everyone becomes the knowledge worker [7].

Knowledge sharing is not a new topic in organization. Current research focuses on the use of formal ontologies for specifying content-specific agreement for a variety of knowledge sharing activities [11]. It is well known that the most valuable knowledge within an organization is not limited to the formal documents in the databases and repositories, but also includes the undocumented ideas, insights and know-how of its members [3]. However, this informal and tacit knowledge is deeply rooted in the individual experience and the culture of work community. To solve these problems, many inventions and solutions emerge, which propose to exploit the embodied knowledge in practice and transform them to a normal form so that other staff and organizations can utilize it. However, it seems that knowledge sharing within organizations is in hot water. The problems are listed as follows: insecure personal identity, no mandate, sanction, time, recognition or reward, poor communication channels, a lack of forms or strict control over agenda and meeting attendance, a culture that regards talking and conversation as "play", cohorts and fellow workers that do not listen, engage or reciprocate, lack of experimentation, risk taking and experience with facilitation and dialog practices. [5]

The objective of this paper is to find reasons why knowledge sharing is difficult to execute within the organization and implement a plan to redesign a new knowledge sharing methodology to solve these problems. Thus, in the following sections we will review the available knowledge sharing theories, limitations and technologies, present the new definition of knowledge sharing based on the past researches support, the research objective and the methodology as well as the work-inprogress status of our research.

\section{LITERATURE REVIEW}

In this section, we will review the literature regarding knowledge sharing and knowledge sharing tools.

\section{A. Knowledge Sharing}

There are not many definitions about knowledge sharing due to the reason that most people see knowledge sharing as a common behavior among organizations and thus neglect its definitions. However, many researchers have recently started to recognize the importance of defining knowledge sharing as a theoretical basis under activities. Firstly, Cummings stated that knowledge sharing involves the process through which knowledge is channeled between a source and a recipient and its meaning within an organization as an organization obtains access to its own and other organization's knowledge [4]. Furthermore, Nelson, R. \& Rosenberg believed that knowledge sharing involves extended learning process rather than simple communication process. [17] From the UTS (University of Technology Sydney) website, it can be known that KS (knowledge sharing?) ensures that people have just-in-time access to the latest information, and that communications systems are specialized in ways that make it easy to use this information [1]. In 1999, Gurteen stated his own idea on knowledge sharing "some people object to sharing as they feel that others will steal their ideas and reap the rewards rightly theirs. This is a fallacy. Knowledge sharing isn't about blindly sharing everything; giving away your ideas; being politically naïve; or being open about absolutely everything. You still need to exercise judgments." [13] In 2003, Cummings defined the overall setting in knowledge sharing, namely five contexts which affect knowledge sharing, as depicted in Fig. 1, which are environmental context, source context, relational context, recipient context and 


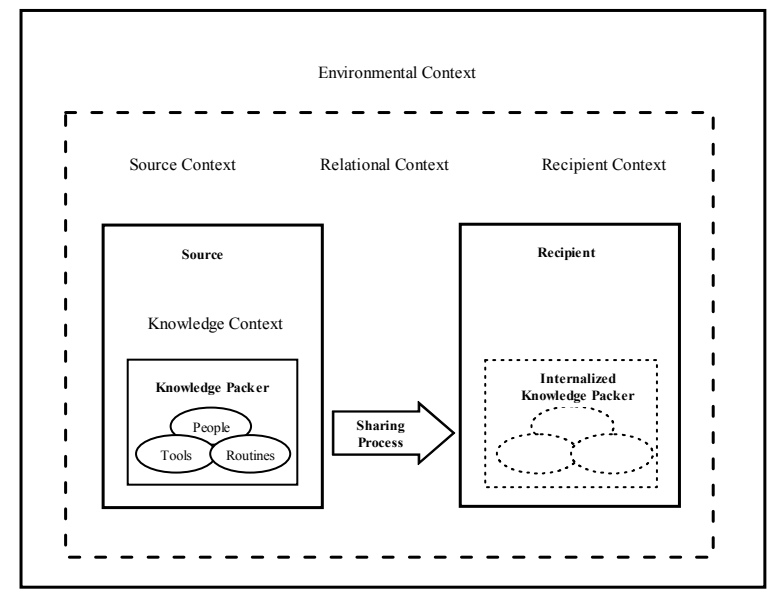

Figure 1. Five contexts of knowledge sharing

knowledge context [4]. Subsequently, Tserng and Lin stated that knowledge sharing enables people to share knowledge and information which is stored in systems by using internet and intranet as well as to exchange explicit and tacit knowledge. In their article, the authors divided knowledge sharing into five steps, which were: search knowledge, refer knowledge, modify knowledge, apply knowledge and collect knowledge. [22].

The above ideas were intended to interpret the definition of knowledge sharing, its scope, process and objectives. Cummings summarized three types of main knowledge sharing activities, including those focused on accessing the form and embeddedness of the knowledge, those focused on establishing and managing an administrative structure through which differences and issues between the parties can be accommodated and reduced and those focused on transferring knowledge [4].

\section{B. Knowledge Sharing Technologies}

With the increasing interest in knowledge sharing, many researchers have started to develop knowledge sharing technologies. Meanwhile many organizations have already been implementing knowledge sharing technologies.

In 1992 Genesereth \& Fikes developed Knowledge Interchange Format (KIF) Version 3.0, which is a computer-oriented language for the interchange of knowledge among disparate programs. It has declarative semantics (i.e. the meaning of expressions in the representation can be understood without appeal to an interpreter for manipulating those expressions); it is logically comprehensive (i.e. it provides for the expression of arbitrary sentences in the first-order predicate calculus); it provides for the representation of knowledge about the representation of knowledge; it provides for the representation of non-monotonic reasoning rules; and it provides for the definition of objects, functions, and relations [10].

In the same year, Finin et al. introduced Knowledge Query and Manipulation Language (KQML) [9]. KQML is a language that programs can use to describe a variety of different attitudes about information including queries, assertions, action requests, information subscriptions, and processing capabilities. Furthermore, KQML is an enabler of information-flow architectures, though forwarding, broadcasting, and brokering messages.
In 1993 McGuire et al. developed the SHADE (SHAred Dependency Engineering) project, which strikes a balance between these undesirable extremes by supporting reconfigurable exchange of engineering knowledge among special-purpose CAE systems. The SHADE approach has three main components: a shared knowledge representation (language and domain-specific vocabulary), protocols supporting information exchange for change notification and subscription, and facilitation services for content-directed routing and intelligent matching of information consumers and producers [15].

According to Merali and Davies, the Knowledge Sharing Environment (Jasper II) is a system of information agents for organizing, summarizing and sharing knowledge from a number of a number of internal and external sources, including the World Wide Web [16]. Jasper II consists of a system of intelligent software agents that retrieve, summarize, and inform other agents about information considered to be of some value by a Jasper II user. The features of KSE include storage and organization of information, matching and selection of information as well as dissemination and delivery.

In 1999, Robertson and Reese introduced an online corporate library in U S WEST library that places knowledge sharing and community building at the core of its design [19]. The library system supports personal websites that are visible to the entire organization. Personal topic profiles for library research services, information services choice and collaborative research requests provide employees with views of each other's activities and interests. In particular, information about research questions being across al parts of the organization provides a unique window on the company's goals and activities. The features of digital library include capture of "ephemeral" information that is usually lost (e.g. conversations), explicit relationships and easy navigation among related pieces of information in disparate sources, widespread availability of information and enabling connections between people who would otherwise never find each other. Thus, the virtual library can help employees to share information across the organization and to form special interest communities.

In 2000 Majchrzak et al. introduced a collaborative tool-Internet Notebook for knowledge sharing and reusing in the knowledge repository [14]. Furthermore, the authors support a mechanism for creating a knowledge repository and a mechanism for accessing the knowledge repository. The notebook allowed team members to access a project knowledge repository, which was housed on a centralized server located at the tool vendor's site. The application provides people with both the knowledge repository as well as such useful capabilities that permitted entries in the notebook, storing entries by data, keyword or reference links, navigating to find entries, creating sketches using a whiteboard, "snapshotting" and hot-liking screen displays from other applications, creating a personal profile for email notification of relevant entries, using templates for frequent team activities, and vaulting documents requiring configuration control.

Process Specification Language (PSL), developed at the National Institute for Standards and Technology, is an ontology that is endorsed as an International Standard within the International Organization of Standardization 
(ISO) [12]. PSL was designed to facilitate correct and complete exchange of process information among manufacturing systems such as scheduling, process modeling, and process planning. The designers of PSL have developed it as an Interlingua for ontologies representing these different processes. All theories within the PSL ontology have been verified with respect to the intended semantics of their terminology. Gruninger and Kopena developed integration architecture with the PSL ontology at the centre and mappings between ontologies for specific manufacturing processes and the PSL ontology. The mappings are defined semi-automatically by presenting ontology developers with a set of questions (in natural language) helping them to map terms in their process specific ontology to the terms in PSL. The system then generates two-way mappings between the taskspecific ontology, such as scheduling and the PSL Interlingua. The generation of these mappings is defined formally and is not based on heuristics. These mappings can be composed to provide mappings between any taskspecific ontologies.

\section{ISSUES IN KNOWLEDGE SHARING ACTIVITIES AND KNOWLEDGE SHARING TECHNOLOGIES}

Sharing knowledge is always a critical topic among organization on account of its impact. Denning presents two drawbacks of knowledge sharing. Firstly, sharing knowledge could lead to the possibility that sharing is insufficiently interactive, and the existence of knowledge sharing implies that the existence of knowledge precedes the sharing process, thereby separating knowledge management from "innovation" and "research" [6].

In addition, for these knowledge sharing technologies, drawbacks are concluded as follows:

Firstly, there is no single knowledge representation that is best for all problems, nor is there likely to be one choice. One form of knowledge representation over another can have a big impact on a system's performance. Even if the representation problems are resolved, it can still be difficult to combine two knowledge bases or establish effective communications between them. The absence of a shared vocabulary presents a further barrier, which could be removed through the development of ontology [8].

\section{KNOWLEDGE SHARING DEFINITION, RESEARCH OBJECTIVE AND SIGNIFICANCES}

\section{A. Definition of Knowledge Sharing in the Context of Organizational Environment}

After reviewing the related literature of knowledge sharing, the new concept of knowledge sharing within an organization based shall be considered as:

Knowledge sharing within an organization is the process of translating the personalized knowledge into the collective knowledge, which consists of knowledge discovery, knowledge extraction and knowledge transfer.

\section{B. Research Objective}

For this research we will address the question below:

'How knowledge sharing within organizations can be enhanced by using semantic web technologies?'
This research will specifically answer following questions:

- What issues affect knowledge sharing within a virtual organization?

- What defects exist in the contemporary knowledge sharing technologies?

- What functionalities can knowledge management systems support for knowledge sharing within a virtual organization?

- What ontology can support knowledge sharing?

- How do we implement the prototype of knowledge sharing technology?

\section{Research Significance}

This research will focus on the theory of knowledge sharing and the development and application of knowledge sharing technologies. The benefits and contribution of this research are shown as follow:

- Provides an alternative knowledge of sharing user interface to assist the communication in organization. It is practical because all of the functional components of the proposed functionalities are based on the existing supporting technologies, rather than in concept.

- Identifies the functionalities that can be enhanced by applying knowledge sharing technologies.

- Involves intensive literature on knowledge sharing, its advantages and disadvantages, which can enable organization with better understanding of knowledge sharing technologies.

- Raises awareness in the organization of the importance of systematically sharing knowledge with colleagues across functions and boundaries in order for organization to be able to produce excellent results and to maintain its advantages in the highly competitive business environment.

- Contributes to strategies and methods of using knowledge sharing within organizations.

It is clear from the literature review that while many theories exist in knowledge sharing, little empirical work has been undertaken. Hence, there is a large gap in the body of knowledge in this area [2]. More researches are needed on knowledge sharing functionalities, reducing knowledge exchange and representation barriers. Theoretically, knowledge sharing technologies should be eligible to enhance knowledge sharing and solve the knowledge exchange barriers to a certain extent. So this research will be based on the knowledge sharing functionalities and the use of knowledge management system.

\section{RESEARCH APPROACH}

The scientific and engineering research method is chosen as our research approach, considering that we propose to develop a knowledge sharing platform and a solution for the problems in knowledge sharing within organizations. Based on the understanding of ontologies and knowledge sharing through the literature review, this research begins with an analysis of which functionalities or components of a knowledge sharing system will have. This section is to select both existing and additional architectural patterns and styles that induce identified 


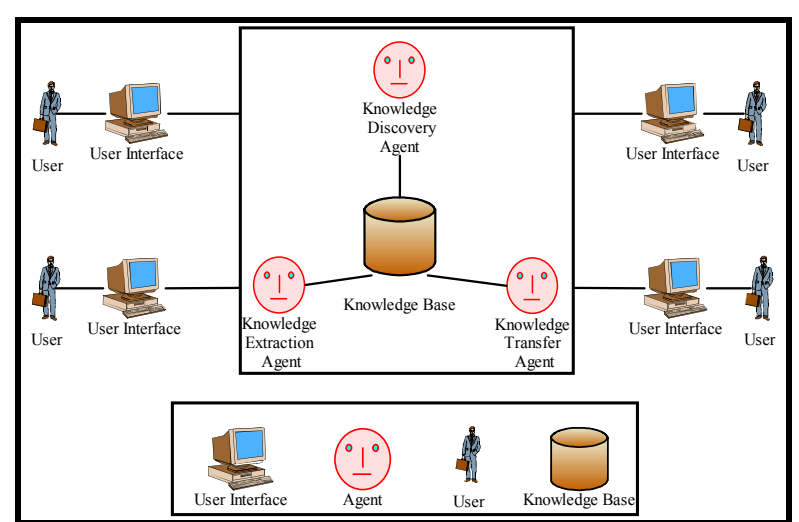

Figure 2. Architecture of proposed platform

properties, and combine them to form a new knowledge sharing system architecture pattern/style. Fig. 2 above illustrates the architecture of knowledge sharing platform drafted in the current phases of our research. The core part of this system is a knowledge base based on an organizational knowledge sharing ontology. Around the knowledge base, we will design multiple agents which execute the function of knowledge discovery, knowledge extraction and knowledge transfer. Finally, a web-based user interface is being proposed for enhancing the knowledge sharing activities in organizations, by allowing users to input data, and share knowledge.

\section{WORK-IN-PROGRESS STATUS}

Currently our work focuses on the construction of a multi-site organizational project knowledge sharing ontology, by means of Protégé-owl. We choose the City of Melville Council in the state of Western Australia - a typical multi-site project organization as the research environment, and design an organizational knowledge sharing ontology based on its organizational project team knowledge (Fig. 3). We make a general survey to the environment and then study the scenarios of knowledge sharing activities in the organization. Based on the ontology, we construct the knowledge base. Next, we develop a query tool based on SPARQL query language, in order to assist the project teams and team members to query project knowledge. Also, a CCCI Metrics, which was developed by Chang and Dillon in 2005, with the purpose of measuring the trustworthiness service, and the reputation of service provider [24], is utilized for team members to evaluate project quality, with the purpose of promoting knowledge sharing between multi-site project teams.
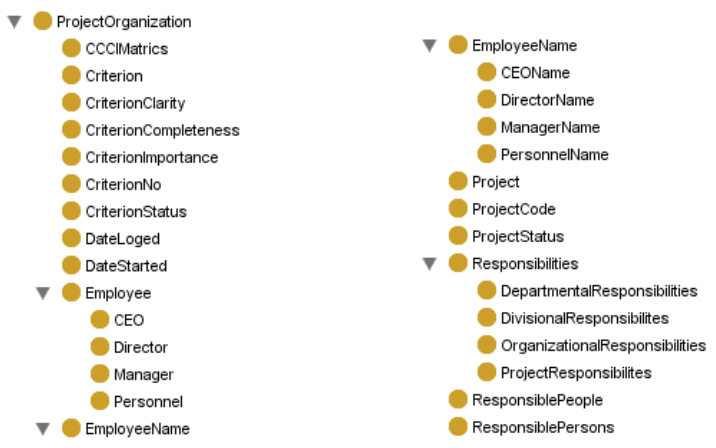

Figure 3. Organizational knowledge sharing ontology in Protégéowl

\section{CONCLUSION AND FURTHER WORKS}

In this paper, we propose an ontology-based knowledge sharing platform, in order to solve the issues in knowledge sharing activities within organizations. Based on the analysis of current literature in this field, we find the issues within the researches with regard to knowledge sharing and knowledge sharing technologies. Also, to conclude the various definitions regarding "knowledge sharing", we provide our understanding towards this term. Against these issues, we provide our research objective, which is to design an ontology-based conceptual model of knowledge sharing platform. We also present the potential contributions of this research. Following that we state the progress of our current research work.

In our further works, we will continue the research towards the design of multiple knowledge sharing agents and web interface for promoting knowledge sharing activities within organizations.

\section{ACKNOWLEDGMENT}

We would like to express our gratitude to the assistance of all DEBII staff.

\section{REFERENCES}

[1] "Knowledge based organizations," Sydney: University of Technology, Sydney, n.d.

[2] M. Alavi and D. E. Leidner, "Review: Knowledge management and knowledge management systems: Conceptual foundations and research issues," MIS Quarterly, vol. 25, pp. 107-136, 2001.

[3] D. G. Bobrow and J. Whalen, "Community knowledge sharing in practice: the EureKa story," Journal of the Society for organization learning and MIT Press, vol. 4, pp. 47-59, 2002.

[4] J. Cummings, "Knowledge sharing: A review of the literature," Washington, D.C.: The World Bank Operations Evaluation Department, 2003

[5] Denham, "Knowledge sharing difficulties \& motivations," 2001.

[6] S. Denning, "What is knowledge management? Definitions," 2004.

[7] P. Drucker, Post-Capitalist Society. New York: Butterworth Heinemann, 1993.

[8] R. Fikes, "Knowledge Sharing Technology Project," Stanford: Stanford University, 1994.

[9] T. Finin, J. Weber, G. Wiederhold, M. Genesereth, R. Fritzson, D. McKay, J. McGuire, P. Pelavin, S. Shapiro, and C. Beck, "Specification of the KQML agent-communication language," Palo Alto: Enterprise Integration Technologies, 1993.

[10] M. R. Genesereth and R. E. Fikes, "Knowledge Interchange Format, Version 3.0 Reference Manual," Stanford: Stanford University, 1992.

[11] T. R. Gruber, "Forward principles for the design of ontologies used for knowledge sharing," International Journal of HumanComputer Studies, vol. 43, pp. 907-28, 1995.

[12] M. Gruninger and J. Kopena, "Semantic integration through invariants," in Workshop on Semantic Integration at ISWC-2003, Sanibel Island: American Association for Artificial Intelligence, 2003.

[13] D. Gurteen, "Creating a knowledge sharing culture," 1999.

[14] A. Majchrzak, R. E. Rice, N. King, A. Malhotra, and S. Ba, "Computer-mediated inter-organizational knowledge sharing: Insights from a virtual team innovating using a collaborative tool," Information Resource Management Journal, vol. 13, pp. 44-53, 2000.

[15] J. G. McGuire, D. R. Kuokka, J. C. Weber, J. M. Tenenbaum, T. R. Gruber, and G. R. Olsen, "SHADE: Technology for knowledge-based collaborative engineering," Journal of Concurrent Engineering: Applications and Research, vol. 1, pp. 137-46, 1993. 
[16] Y. Merali and J. Davies, "Knowledge capture and utilization in virtual communities," in the 1st international conference on Knowledge capture Victoria, 2001, pp. 92-99.

[17] R. Nelson and N. Rosenberg, "Technical innovation and national systems," in National innovation systems: A comparative analysis New York: Oxford University Press, 1993.

[18] S. L. Pan and D. E. Leidner, "Bridging communities of practice with information technology in pursuit of global information sharing," Journal of Strategic Information Systems, vol. 12, pp. 71-88, 2003.

[19] S. Robertson and K. Reese, "A virtual library for building community and sharing knowledge," Int. J. Human-Computer Studies, vol. 51, pp. 663-685, 1991.
[20] J. C. Spender, "Making knowledge the basis of a dynamic theory of the firm," Strategic Management Journal, vol. 17, pp. 45-62, 1996.

[21] A. Stinchcombe, Information and Organizations. Los Angeles: University of California Press, 1990.

[22] H. P. Tserng and Y. C. Lin, "Developing an activity-based knowledge management system for contractors," Automation in Construction, vol. 13, pp. 781-802, 2004.

[23] G. N. von Tunzelmann, Technology and Industrial Progress. Aldershot: Edward Elgar, 1995.

[24] E. Chang, T. S. Dillon, and F. Hussain, Trust and Reputation for Service Oriented Environments-Technologies for Building Business Intelligence and Consumer Confidence: John Wiley \& Sons, 2005. 